\title{
ESTUDO DO COMPORTAMENTO FLUIDODINÂMICO DE CICLONES*
}

\author{
STUDY OF CYCLONE FLUID DYNAMIC BEHAVIOR
}

\author{
ESTUDIO DEL COMPORTAMIENTO FLUIDODINÁMICO DE LOS CICLONES
}

\author{
Aderjane Ferreira Lacerda \\ Reimar de Oliveira Lourenço \\ Paulo Roberto Campos Castro Filho
}

\begin{abstract}
Resumo: Objetiva-se avaliar o comportamento fluidodinâmico de ciclones através do uso de técnicas numéricas utilizadas em CFD (Computational Fluid Dynamics). Utilizou-se como variáveis de entrada dados experimentais conhecidos. Adotou-se os seguintes modelos de turbulência para compará-los entre si: modelo k- $\varepsilon$; modelo RSM (Reynolds Stress Model). O fluido utilizado nas simulações foi o ar e as partículas adicionadas foram de carbonato de cálcio $\left(\mathrm{CaCO}_{3}\right)$. Os parâmetros estudados foram queda de pressão, perfis de velocidade e a eficiência de coleta do ciclone. O modelo de turbulência adotado que apresentou melhores resultados foi o RSM. A queda de pressão para o RSM foi da ordem 868,6 Pa e para o k- $\square$ de $758 \mathrm{~Pa}$, para o modelo misto (k- $\square$ / RSM) de $836 \mathrm{~Pa}$ e para os resultados experimentais realizados por Patterson e Munz de $1100 \mathrm{~Pa}$. Os perfis de distribuição de velocidade (tangencial, axial e radial) e de pressão apresentaram boa concordância com os dados da literatura, maiores velocidades encontram-se entre a parede do equipamento e seu eixo de simetria. Para os perfis de pressão, eles apresentaram maiores pressões nas paredes e menores próximas ao eixo. Utilizou-se o modelo de distribuição de RosinRammler para avaliar a eficiência de coleta do ciclone, observando-se que, para partículas de diâmetro superior ao Diâmetro de Corte das Partículas $\left(d_{p c}\right)$ do equipamento, a eficiência tende a ser superior a 50\%, aumentando à medida que estes aumentam, e para diâmetros inferiores, inferior a 50\%. A utilização das técnicas de CFD para caracterização do escoamento gasoso em ciclones permitiu obter resultados bastante coerentes quando comparados com dados experimentais da literatura.
\end{abstract}

Palavras-chave: Ciclones. Separação gás-sólido. CFD.

Abstract: The objective is to evaluate the fluid dynamic behavior of cyclones through the use of numerical techniques used in CFD (Computational Fluid Dynamics). It is used as input variables known experimental data. We adopted the following turbulence models to compare them to each other: $k-\varepsilon$ model; model RSM (Reynolds Stress Model). The fluid used in the simulations was the air and the particles added were calcium carbonate $\left(\mathrm{CaCO}_{3}\right)$. The parameters studied were pressure drop, velocity profiles and the collection efficiency of the cyclone. The turbulence model adopted that showed the best results was the RSM. The pressure drop for the RSM was approximately $868.6 \mathrm{~Pa}$ and the $\mathrm{k}-\square$ of $758 \mathrm{~Pa}$ for the mixed model (k- $\square$ / RSM) to $836 \mathrm{~Pa}$ and the experimental results performed by Patterson and Munz $1100 \mathrm{~Pa}$. distribution profiles of velocity (tangential, axial and radial) and pressure were in good agreement with the literature data, higher speeds lying between the wall of the equipment and the symmetry axis thereof, the pressure profiles for the they showed higher pressures and lower walls near the axis. We used the model Rosin-Rammler distribution to evaluate the collection efficiency of the cyclone, observing that for particles of diameter greater than the cutting diameter $\left(d_{p c}\right)$ equipment efficiency tends to be higher than $50 \%$, increasing as these increases, and smaller diameters of less than $50 \%$. In general, the use of CFD techniques for characterizing the gas flow in cyclone yielded fairly consistent results when compared to experimental data in the literature.

Keywords: Cyclones. Gas-solid separation. CFD.

Resumen: El objetivo es evaluar el comportamiento dinámico de fluido de los ciclones a través del uso de técnicas numéricas utilizadas en CFD (Computational Fluid Dynamics). Se utiliza como variables de entrada los datos experimentales conocidos. Hemos adoptado los siguientes modelos de turbulencia para compararlos entre sí: $k-\varepsilon$ modelo, modelo RSM (Modelo Reynolds Stress). El fluido utilizado en las simulaciones era el aire y las partículas añadidas fueron de carbonato de calcio $\left(\mathrm{CaCO}_{3}\right)$. Los parámetros estudiados fueron la caída de presión, velocidad y perfiles de la eficiencia de recogida del ciclón. El modelo de turbulencia adoptado, y que mostró los mejores resultados fue la RSM. La caída de presión para el RSM fue de aproximadamente 868,6 Pa y el k- $\square$ de 758 Pa para el modelo mixto (k- $\square$ / RSM) a 836 Pa y los resultados experimentales realizados por Patterson y Munz 1100 Pa. Los perfiles de distribución de velocidad (tangencial, radial y axial) y la presión estuvieron en buen acuerdo con los datos de la literatura, velocidades más altas situadas entre la pared del equipo y el eje de simetría de los mismos, los perfiles de presión para la mostraron altas presiones y bajas paredes cerca del eje. Se utilizó el modelo de distribución de Rosin-Rammler para evaluar la eficacia de recogida del ciclón, la observación de que para las partículas de diámetro mayor que el diámetro de corte $\left(d_{p c}\right)$ la eficiencia del equipo tiende a ser mayor que $50 \%$, aumentando también cuando ellos aumentan y para diámetros más pequeños, de menos de $50 \%$. En general, el uso de técnicas de CFD para la caracterización del flujo de gas en el ciclón dió resultados bastante consistentes cuando se compara con los datos experimentales en la literatura. Palabras clave: Ciclones. Separación gas-sólido. CFD.

Trabalho premiado durante o XXIV Encontro do SEMIC, realizado na UFMA entre os dias 05 a 08 de novembro de 2012.

*Artigo recebido em dezembro 2012

Aprovado em fevereiro 2013 


\section{INTRODUÇÃO}

Atualmente existe uma grande preocupação com a poluição do ambiente. Com base neste fato, foram criadas diversas leis e protocolos para que as emissões de compostos poluentes fossem diminuídas até um ponto aceitável. Como exemplo, para o caso de emissão de partículas no ar, no Brasil, pode-se citar a resolução de no 3 do CONAMA, datada de $28 / 06 / 1990$, estabelecendo que os limites exigidos para a emissão de partículas inaláveis (diâmetro < $10 \mu \mathrm{m}$ ) num padrão primário (nível máximo) são de $240 \mu \mathrm{g} / \mathrm{m} 3 e$ secundário (nível desejável) são de $150 \mu \mathrm{g} / \mathrm{m} 3$.

Os ciclones são equipamentos muito utilizados, antigos e conhecidos usados para separação de partículas sólidas presentes em correntes gasosas. Constituem-se basicamente de uma região cilíndrica acoplada a uma seção cônica. (LACERDA, 2007).

Podem ser empregados em indústrias químicas, metalúrgicas, alimentícias e na área ambiental, onde tem maior importância. Os ciclones, atualmente, estão sendo utilizados em novos processos como secadores, reatores e recuperadores catalíticos onde há alto valor agregado.

O princípio pelo qual as partículas são removidas da fase fluida ocorre pela ação do campo centrífugo resultante do equipamento. Inicialmente realiza um movimento circulatório na seção anular compreendida entre o orifício superior (overflow) e o corpo do ciclone, provocando a formação de um vórtice descendente. Esse movimento em espiral estende-se até a base do ciclone. As partículas presentes na corrente gasosa, devido à ação da força centrífuga, deslocam-se em direção à parede do ciclone, o que faz com que elas percam velocidade por atrito e tendam a escorregar pelas paredes, sendo coletadas por um recipiente, no orifício inferior (underflow). O gás, por sua vez, ao se aproximar do final da parte cônica, inverte o seu sentido, dirigindo-se à saída superior do ciclone, o que produz um vórtice interno ascendente, conforme detalhe na figura 01 . O gás que deixa o ciclone, geralmente, possui uma baixa concentração de partículas, sendo estas mais finas (LACERDA, 2007).

Diversos trabalhos foram produzidos sobre ciclones, por este motivo existem diversas famílias de ciclones que são amplamente utilizadas devido ao grande número de estudos já produzidos sobre estes equipamentos. Alguns trabalhos já executados buscavam a relação entre as diferentes geometrias existentes e sua eficiência na coleta de partículas; outros visavam avaliar a relação entre diferentes velocidades empregadas e a perda de carga, juntamente com a eficiência de coleta.

Atualmente modelos matemáticos que tratam de um conjunto de equações diferenciais parciais não-lineares que governam a conservação de massa, quantidade de movi- mento e energia, os quais descrevem variáveis em geometrias bidimensionais e tridimensionais, estão se tornando cada vez mais objeto de estudo. Dessa maneira, a simulação com técnica da fluidodinâmica computacional Computational Fluid Dynamics (CFD) surgiu como uma solução econômica e eficaz na predição de fenômenos relacionados com o escoamento em ciclones, o qual se baseia nos princípios de conservação de massa, de quantidade de movimento e de energia para escoamento dos fluidos. Com isso essa técnica tem sido utilizada para resolver equações não-lineares que descrevem o escoamento dos fluidos, além de modelar ciclones com diferentes níveis de complexidade, podendo tratar vários aspectos operacionais relacionados a esse equipamento, como a reversão de fluxo, a alta vorticidade, as zonas de circulação e o fluxo descendente (CARVALHO et al., 2012).

Figura 1 - Trajetória da mistura gás-sólido no interior do ciclone

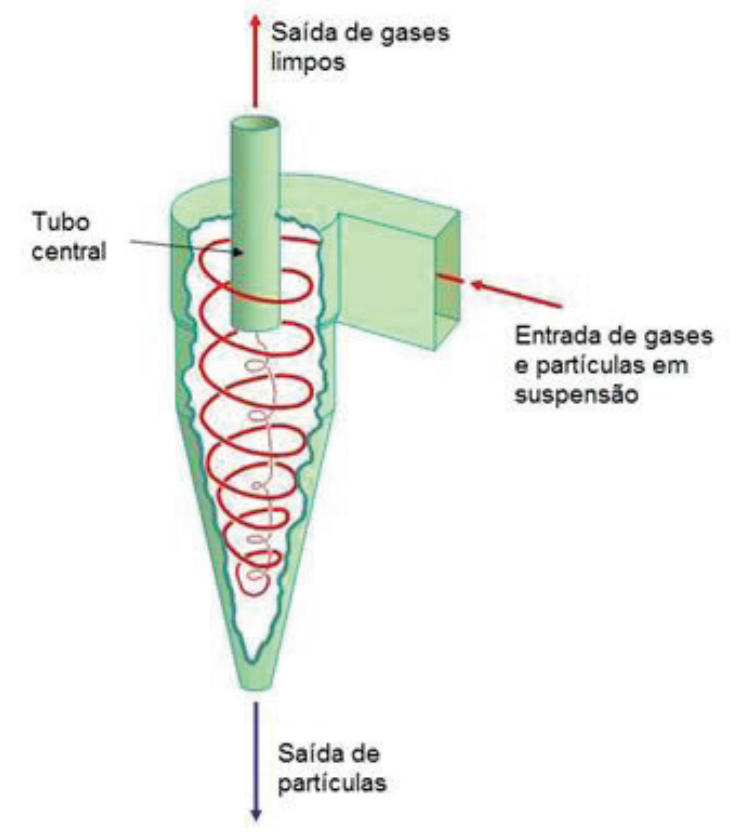

Fonte: Ciclones... (2012)

A partir disso, realizar-se-á um estudo computacional sobre as principais variáveis de projeto de um ciclone através de simulações para identificar a consistência de modelos computacionais frente a modelos empíricos e, dessa forma, avaliar o uso de sistemas computacionais em detrimento de testes experimentais.

\section{METOdOLOGIA DE SIMULAÇÃo}

Para esta etapa de pré-processamento, definiu-se primeiramente as dimensões para o ciclone do tipo Lapple utilizado para geração de malhas bidimensionais, conforme descrito na Tabela 2. 
Figura 2 - Estrutura de um Ciclone Convencional

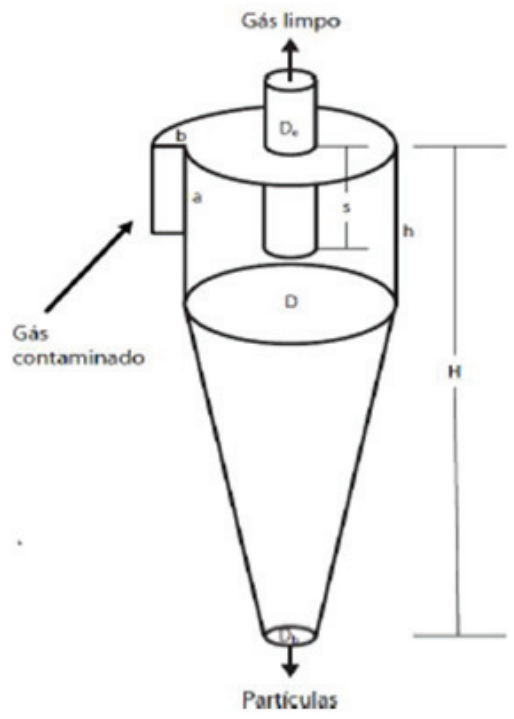

Fonte: Adaptado de Carvalho et al. (2012)

Tabela 1 - Dimensões do Ciclone Simulado

\begin{tabular}{c|c}
\hline Dimensão do Ciclone Lapple & Dimensões \\
\hline Diâmetro do ciclone (Dc) & $0.0120 \mathrm{~m}$ \\
\hline Diâmetro do duto de saída do gás (De) & $0.0508 \mathrm{~m}$ \\
\hline Altura (interior) do finder (S) & $0.108 \mathrm{~m}$ \\
\hline Altura da parte cilíndrica (h) & $0.203 \mathrm{~m}$ \\
\hline Somatório das alturas do cilindro \\
e cônica (H) & 0.406 \\
\hline Diâmetro da parte inferior do cone (Db) & $0.0254 \mathrm{~m}$ \\
\hline Altura da entrada (a) & $0.0508 \mathrm{~m}$ \\
\hline Altura do coletor de pó (c) & $0.102 \mathrm{~m}$ \\
\hline Diâmetro do coletor de pó (Dco) & $0.102 \mathrm{~m}$ \\
\hline Altura do cone (Z) & $0.203 \mathrm{~m}$ \\
\hline
\end{tabular}

Fonte: Elaborado pelos autores

\subsection{Geração de malha}

A precisão da simulação numérica do ciclone é função da qualidade da malha, pois esta representa o domínio de cálculo onde as equações de Navier-Stokes discretizadas serão resolvidas numericamente. A malha deve ficar refinada dentro do ciclone e particularmente perto das paredes nas superfícies cônicas e cilíndricas, onde, teoricamente, há fortes gradientes de velocidade, queda de pressão e captura do material particulado (RAMIREZ, 2009).

A construção da malha é orientada pela escolha de um referencial no plano xy. Assim, apenas uma das partes que compõem a simetria será construída, desde que obedecidas algumas exigências impostas pelo software. A primeira dela refere-se ao eixo de simetria do equipamento, o qual deve coincidir com o eixo $x$. A outra diz respeito ao domínio da malha: ele deve estar situado numa região cujos valores de y sejam positivos. Desta maneira, os demais extremos do sistema são definidos de forma pontual pelo fornecimento das respectivas co- ordenadas $(x, y)$. Definidos os principais vortex (vértices), estes são ligados através de segmentos de reta chamados edges (linhas), que representam os tipos de fronteiras existentes no sistema. Um conjunto de edges recebe o nome de faces, nas quais o usuário estabelece o tipo de fluido (monofásico ou mistura) ou sólidos utilizados na simulação. Por fim, um conjunto de faces dá origem aos volumes, os quais representam por inteiro todo o domínio a ser simulado (VIEIRA, 2006). Indicar adequadamente cada elemento do equipamento é um ponto de vital importância para o software simulador devido ao fato de indicar as condições de contorno aplicadas, além das barreiras físicas do equipamento, por exemplo, paredes externas e internas.

Neste trabalho, a malha computacional utilizada é composta apenas de células quadrangulares, devido à maior estabilidade que ela proporciona. Para a construção da malha em determinada face, cada edge recebe um número determinado de pontos, condição de fundamental importância na simulação, visto que um número muito reduzido de pontos irá gerar poucas células na malha final, comprometendo o resultado final. Por outro lado, um número muito grande poderá tornar a simulação muito lenta e inclusive dificultar sua convergência. Para o caso estudado, o número de pontos (células) criados foi de 130767.

Figura 3 - Malha gerada com suas várias linhas de construção

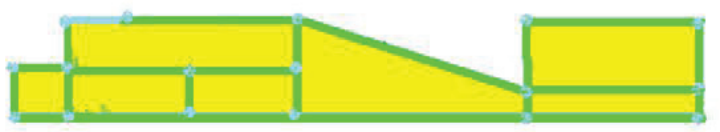

Fonte: GAMBIT 6.2

Atribui-se a cada edge um tipo de fronteira, para que posteriormente estejam aptas a receber as condições de contorno necessárias. A Tabela 2 apresenta as identificações para as edges.

Tabela 2 - Condições de contorno aplicadas no Ciclone

\begin{tabular}{c|c}
\hline Fronteira & Especificação \\
\hline Parede & Wall \\
\hline Entrada & Velocity Inlet \\
\hline Saída (overflow) & Pressure Outlet \\
\hline Linhas internas & Interior \\
\hline Eixo & Axis \\
\hline
\end{tabular}

Fonte: Elaborada pelos autores

\subsection{Condições de contorno}

Após gerar a malha, o passo seguinte é abri-la no simulador onde todos os dados de funcionamento do ciclone devem ser introduzidos, como velocidade de entrada da mistura gás-sólido, os materiais e com este suas ca- 
racterísticas que já se encontram no banco de dados do programa (viscosidade, massa específica, etc). Os materiais usados na simulação foram o ar com massa específica $\left(\rho_{a r}\right)$ de 1,225 $\mathrm{Kg} / \mathrm{m}^{3}$ e viscosidade $(v)$ de $185 \times 10^{-\mathrm{s}} \frac{\mathrm{Kg}}{\mathrm{ms}}$ e as partículas de carbonato de cálcio $\left(\mathrm{CaCO}_{3}\right) \mathrm{com}$ massa específica $\left(\rho_{p}\right)$ de $2800 \mathrm{~K} / \mathrm{m}^{3}$.

Escolhe-se o tipo de injeção, o tipo e o número de partículas, o modelo de distribuição granulométrica, as coordenadas de início e término da injeção, a massa alimentada, os diâmetros mínimo e máximo das partículas, os parâmetros do modelo utilizado e os valores das componentes da velocidade do fluido (axial, radial e tangencial). No caso, a componente axial da velocidade $\left(\mathrm{w}_{\mathrm{e}}\right)$ do fluido na entrada do ciclone é nula, pois o ar é introduzido no separador na direção de seu diâmetro e não no de seu eixo de simetria. Em relação à componente radial da velocidade $\left(u_{e}\right)$ do fluido, esta é calculada com base na transformação teórica da entrada dos ciclones em uma entrada bidimensional simétrica, conforme descrito por Boysan, Ayers, swithenbank (1982). Vale ressaltar que, como a componente radial de velocidade do fluido é uma grandeza vetorial, ela poderá vir acompanhada do sinal negativo, de acordo com a disposição do eixo das ordenadas (VIEIRA, 2006).

$$
U_{e}=\frac{Q}{\pi D_{i} D_{c}}
$$

A componente tangencial ( ) do fluido é calculada, considerando as dimensões exatas do tubo de alimentação do ciclone. Porém, para que esta condição esteja disponível durante as simulações, é necessário mencionar ao programa computacional o fato de que o espaço da simulação é regido pela simetria e por escoamento rotacional do fluido (Axisymmetric Swirl) (VIEIRA, 2006).

$V_{e}=\frac{4 Q}{\pi D_{i}^{2}}$

Em relação às saídas de underflow e overflow, admitiram-se ambas com pressões atmosféricas locais (101325 Pa).

A tabela 3 apresenta de forma resumida os dados aplicados à simulação:

Tabela 3 - Resumo de condições e modelos adotados

\begin{tabular}{c|c}
\hline Tipo de partícula & Inerte \\
\hline Material & $\begin{array}{c}\text { Carbonato de Cálcio } \\
\left(\mathrm{CaCO}_{3}\right)\end{array}$ \\
\hline Velocidade Tangencial $(\mathrm{m} / \mathrm{s})$ & 15,08 \\
\hline Velocidade Radial $(\mathrm{m} / \mathrm{s})$ & $-1,2$ \\
\hline Velocidade Axial (m/s) & 0 \\
\hline $\begin{array}{c}\text { Modelo para Discretização da } \\
\text { Pressão }\end{array}$ & PRESTO \\
\hline
\end{tabular}

\begin{tabular}{c|c}
\hline $\begin{array}{l}\text { Método para Acoplamento } \\
\text { Pressão-Velocidade }\end{array}$ & SIMPLE \\
\hline Momento & Primeira Ordem \\
\hline Modelo de Turbulência & k- $\square / \begin{array}{c}\text { Reynolds Stress } \\
\text { Model }\end{array}$ \\
\hline
\end{tabular}

Fonte: Elaborada pelos autores

\subsection{Modelos}

Quanto aos modelos de turbulência, foi escolhido o modelo RSM (Reynolds Stress Model), devido à complexidade de escoamento no interior dos ciclones. Já em relação à interpolação de pressão, optou-se pelo esquema PRESTO. Quanto ao acoplamento do binômio pressão-velocidade, utilizou-se o algoritmo SIMPLE. Para as demais variáveis fluidodinâmicas, a escolha de esquemas de interpolação foi do tipo UPWIND, sejam de primeira ou segunda ordem (VIEIRA, 2006).

\section{RESULTADOS E DISCUSSÕES}

De acordo com os dados de entrada fornecidos ao software, os resultados da simulação puderam ser obtidos de várias maneiras, devido às diversas ferramentas fornecidas pelo software para visualização de resultados.

\subsection{Queda de pressão}

Apresentam-se a seguir os dados referentes aos modelos de turbulência adotados para as simulações, os quais serão comparados entre si. O software possibilita o cálculo do valor médio de uma grandeza. Na tabela 4 , estão os valores comparativos da queda de pressão, entre a alimentação e a saída de gás, simulados com o modelo de queda de pressão proposto por Shepherd e Lapple (1939).

Tabela 4 - Valores de Queda de Pressão médios para simulações e experimento

\begin{tabular}{c|c|c|c|c}
\hline $\begin{array}{c}\text { Queda de } \\
\text { Pressão (Pa) }\end{array}$ & $\mathrm{k}-\varepsilon$ & $\mathrm{RSM}$ & $\mathrm{k}-\varepsilon / \mathrm{RSM}$ & Empírico \\
\hline Simulado & 758.1 & 868.53 & 836.05 & - \\
\hline Experimental & - & - & - & 1100 \\
\hline
\end{tabular}

Fonte: Elaborada pelos autores

Observa-se que o modelo RSM foi mais eficiente no que se diz respeito à queda de pressão, sendo seu valor o mais próximo ao do experimental. Esta diferença entre o modelo RSM e o empírico pode estar associado ao refinamento da malha, além da simulação ser bidimensional, com isso não retratando de forma eficaz todas as regiões do ciclone.

A Figura 4 mostra os perfis de pressão absoluta, respectivamente para os modelos $k-\varepsilon$, RSM e k- $\varepsilon$ / RSM, o qual apresenta camadas bem definidas de pressão no ciclone, com 
máximas nas paredes e mínimas no eixo. Este comportamento é coerente com os trabalhos de Patterson e Munz (1996).

Figura 4 - Perfis de contornos de pressão

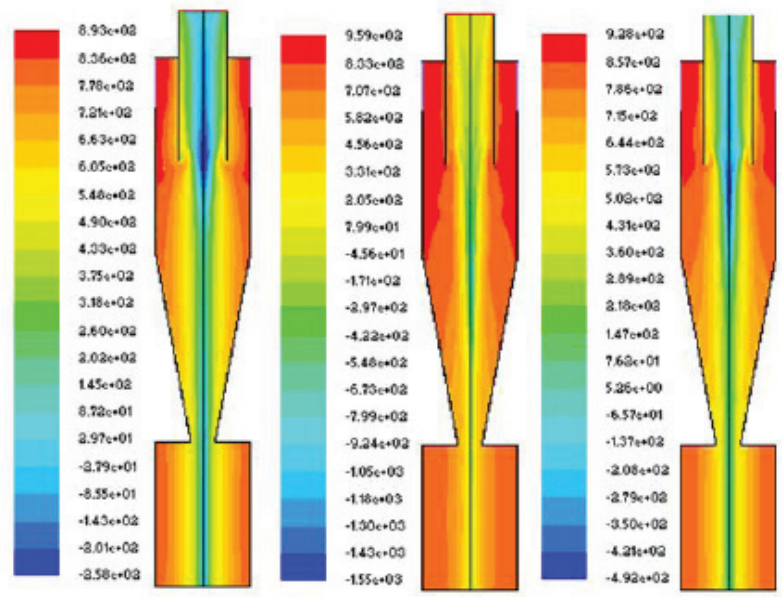

Fonte: FLUENT 6.2

De acordo com os perfis de pressão acima, pode-se notar que a zona de maior pressão esta situada próximo à entrada do aparelho e às suas paredes e que, próximo ao eixo do equipamento (simetria), existe uma zona de baixa pressão, considerada como a de reversão de fluxo, onde o fluxo que era descendente passa a ser ascendente e direciona-se para a saída (overflow).

\subsection{Perfis de velocidade}

Na próxima figura, estão apresentados os contornos de perfil de velocidade tangencial (v) para os modelos na mesma ordem citada anteriormente.

Figura 5 - Perfis de Velocidade Tangencial (v) em $\mathrm{m} / \mathrm{s}$

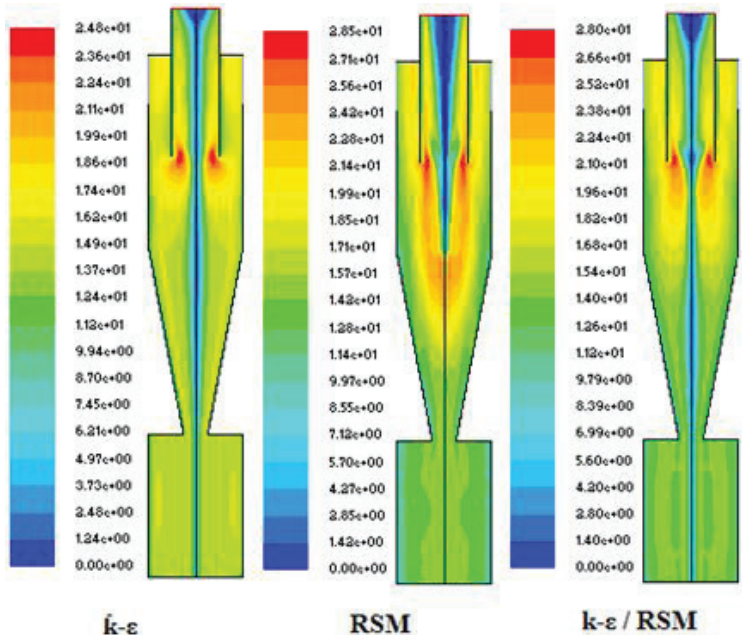

Fonte: FLUENT 6.2

Na figura 6, são apresentados os perfis de velocidade axial ( $W$ ) para cada um dos modelos $k-\varepsilon, R S M$ e $k-\varepsilon /$ RSM, respectivamente.
Figura 6 - Contornos dos Perfis de Velocidade Axial (w) em m/s

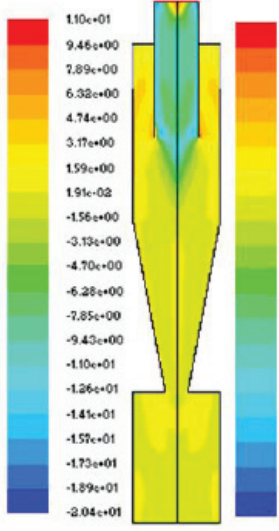

k- $\varepsilon$

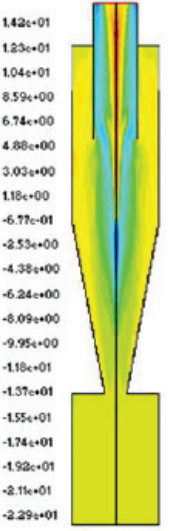

RSM

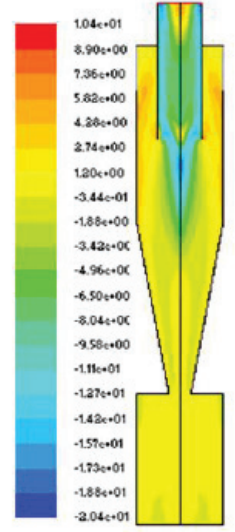

k- $\varepsilon$ / RSM
Fonte: FLUENT 6.2

As Figuras 5 e 6 apresentam os perfis de velocidade tangencial e axial, respectivamente, com dimensões definidas por Patterson e Munz (1996). Foram simulados por Lacerda (2007) e apresentados a seguir:

Figura 7: Perfis de velocidade axial (a) e tangencial (b) para o modelo RSM, com dimensões definidas por Patterson e Munz (1996)

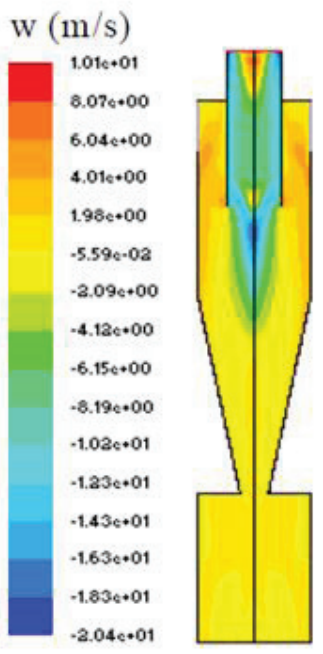

(a)

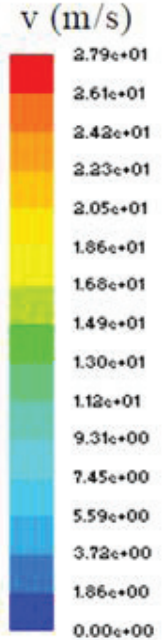

$0.00 \mathrm{c} * 00$

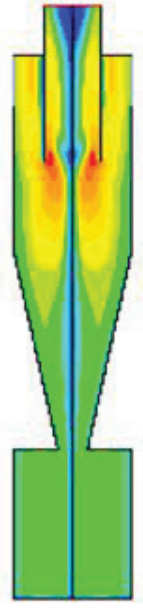

(b)
Fonte: Lacerda (2007).

Observa-se, nas três figuras acima, semeIhanças entre os contornos do modelo RSM. No trabalho realizado por Lacerda (2007), apenas o modelo RSM foi levado em consideração, pois ter apresentado melhor proximidade com os dados experimentais. Em relação ao modelo $k-\varepsilon$, alguns pesquisadores demonstraram que este modelo de turbulência não consegue representar o funcionamento de ciclones. Apesar de ter-se o gradiente radial de pressão próximo às zonas de entrada e a velocidade tangencial acusar que há um movimento de rotação, ele simplesmente não consegue se sustentar ao longo do domínio, devido à inadequação do modelo em prever esta vorticidade (DIAS, 2009). 
No modelo RSM e k- $\varepsilon$ / RSM, nota-se que a velocidade tangencial é baixa na parede e no eixo e apresenta um valor de máximo próximo ao centro, sendo este o comportamento característico para essa velocidade. A velocidade axial indica um fluxo descendente próximo à parede e um fluxo ascendente no rumo do vortex finder, sendo essa uma característica funcional do equipamento.

As figuras 7 e 8 apresentam os perfis de velocidade axial (w) e tangencial (v) do fluido, respectivamente, em função da posição radial (r) para o modelo RSM, que possui os resultados mais relevantes. As linhas representam os perfis de velocidade simulados, onde os perfis foram retratados em um ponto que corresponde a uma altura de 0.13 metros do topo do ciclone, a exemplo do que foi descrito por Patterson e Munz (1996).

Figura 8 - Distribuição de velocidade axial ( ), RSM

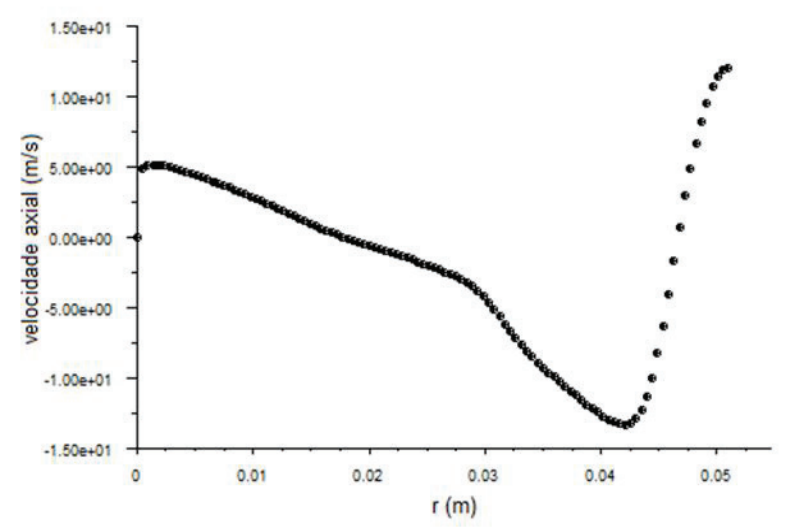

Fonte: FLUENT 6.2

Tem-se pelo componente axial que a velocidade nesse ciclone simulado apresenta um comportamento descendente junto à parede e gradativamente inverte seu sentido no seu eixo de simetria; o gás passa a ir em direção à saída do ciclone, a exemplo dos resultados apresentados por Patterson e Munz (1996).

Figura 9 - Distribuição de velocidade tangencial (v), RSM

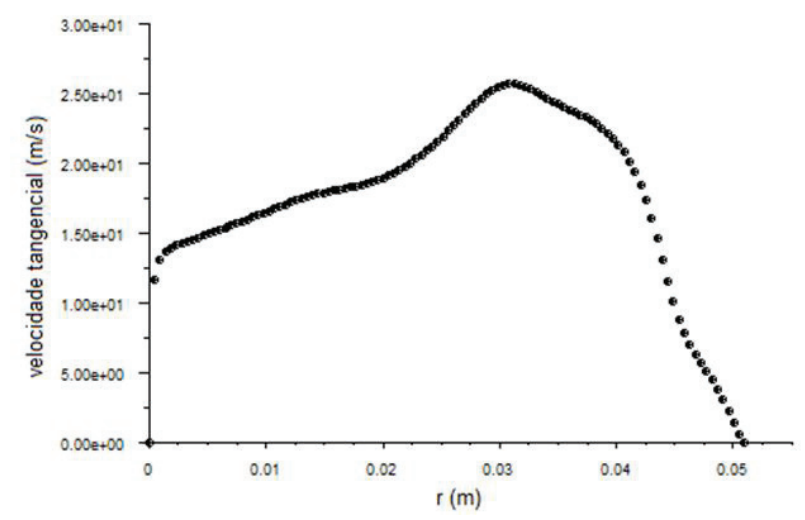

Fonte: FLUENT 6.2
Patterson e Munz (1996) afirmam que a velocidade tangencial inicialmente aumenta da parede para o centro, assumindo um valor máximo, quando então decai rapidamente. O que ocorreu de forma equivalente para o modelo RSM.

Em relação às três simulações realizadas, pode-se constatar que o modelo RSM foi o único capaz de representar o fenômeno que ocorre dentro de um equipamento de separação gás-sólido do tipo ciclone, sendo que o modelo $k-\varepsilon / R S M$ não é de utilidade, pois, mesmo usando de dois modelos combinados, ainda assim o modelo RSM apresentou melhores resultados.

\subsection{Avaliação da trajetória de partículas e eficiência}

Para o processo de verificação da trajetória das partículas e eficiência de coleta, foi adicionado um grupo de 100 partículas de carbonato de cálcio $\left(\mathrm{CaCO}_{3}\right)$ na entrada do ciclone, que foi especificado através das coordenadas da entrada do equipamento atribuídas durante a geração da geometria, e novamente adicionando as velocidades de funcionamento do equipamento (velocidade tangencial, axial e radial). O modelo de distribuição adotado foi o modelo de Rosin-Rammler, que é a utilização de distribuição probabilística para a previsibilidade da partição de fluxos de particulados em equipamentos de processos

Para verificação da eficiência de coleta do equipamento, calculou-se o diâmetro de corte para este ciclone, através da equação abaixo:

$$
d p c=\left(\frac{9 \mu a}{2 \pi N v(\rho s-\rho)}\right)
$$

onde:

- $\mu$ é a viscosidade do gás;

- a é a largura da entrada do ciclone;

- Ne é o número de voltas que o gás executa no interior do ciclone na espiral descendente. Nesse caso para o ciclone Lapple, $\mathrm{N}$ é igual a 5 , medida a qual foi determinada experimentalmente;

- $v$ é a velocidade do fluido no duto de entrada do ciclone. De acordo com Massarani (2002), a velocidade recomendada situa-se na faixa de 6 a $21 \mathrm{~m} / \mathrm{s}$, sendo de 15,2 m/s a velocidade recomendada;

- $\rho s$ e $\rho$ são as massas específicas do sólido e do gás, respectivamente.

O diâmetro de corte é um parâmetro que está relacionado à eficiência de coleta. Este parâmetro depende das propriedades do sólido, das propriedades do gás, do tamanho do ciclone e das condições operacionais. Uma definição simples associa o diâmetro de corte ao tamanho da partícula que é coletada com $50 \%$ de eficiência. Com isto as partículas que possuem os diâmetros maiores que o diâmetro de corte são coletados no underflow com efici- 
ências maiores que 50\% (LACERDA, 2007). O valor do $d_{p c}$ calculado pela equação acima foi de aproximadamente $2,45 \mu \mathrm{m}$. Tal valor foi a base para a simulação da trajetória das partículas.

As Figuras 10 e 11 apresentam as simulações da trajetória de 100 partículas distribuídas em diferentes diâmetros ao longo do ciclone. Tais diâmetros propostos estão abaixo e acima do $\mathrm{d}_{\mathrm{pc}}=2,45 \mu \mathrm{m}$, respectivamente, para os três modelos de turbulência propostos.

Figura 10 - Diâmetro das partículas menor que o diâmetro de corte

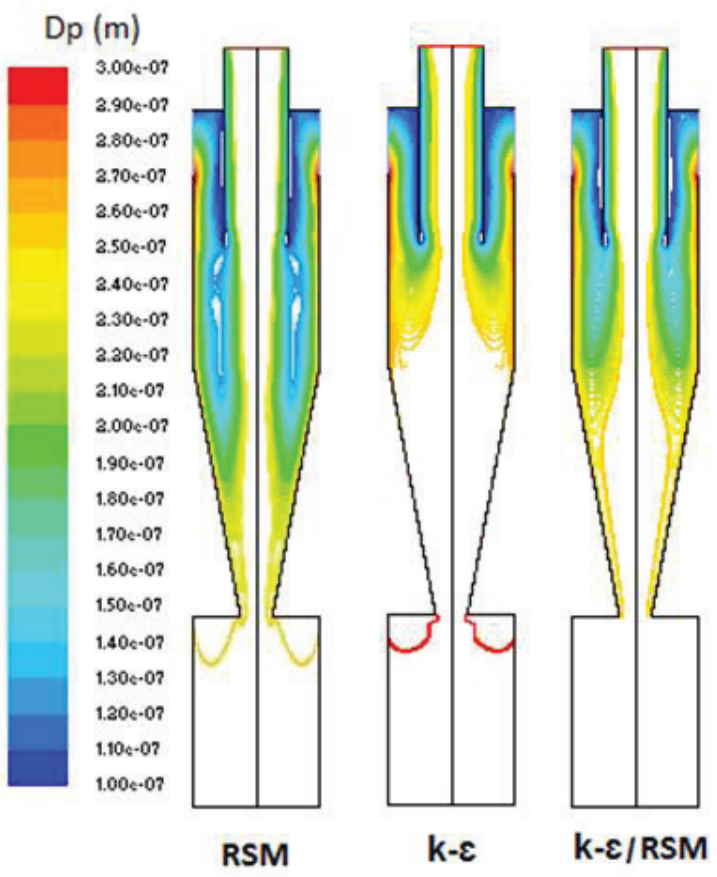

Fonte: FLUENT 6.2

Figura 11 - Diâmetro das partículas entre o diâmetro de corte

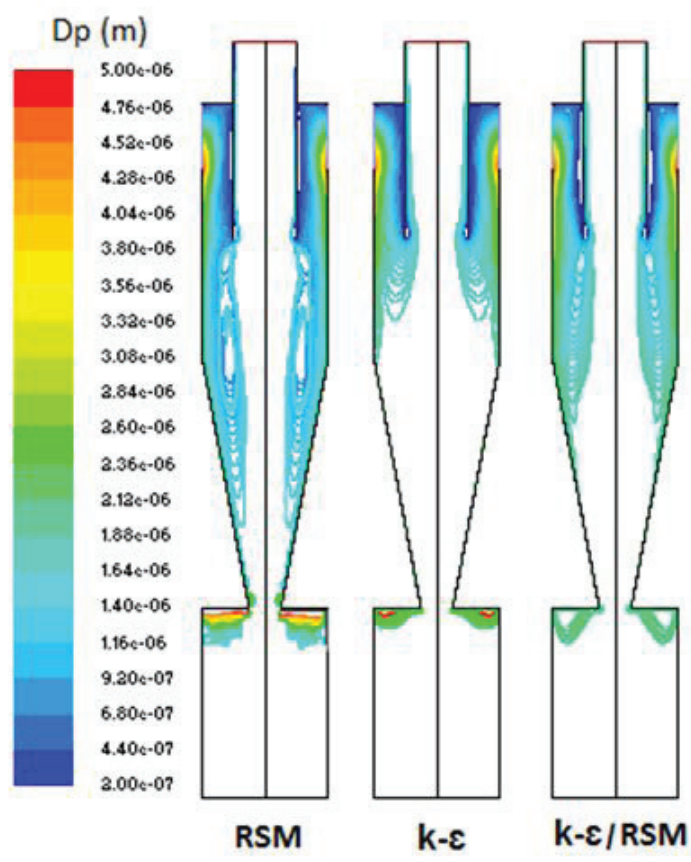

Fonte: FLUENT 6.2
De acordo com as figuras acima, verifica-se que as partículas de maiores diâmetros em ambos os casos encontram-se mais próximas à parede do equipamento, assim como encontrado na literatura, as partículas de diâmetros intermediários prosseguem sua trajetória descendente. Algumas destas são coletadas e outras se direcionam para a saída superior do equipamento, passando antes por zonas de recirculação, como é possível perceber em todos os casos.

Em relação à figura 10 , verifica-se que novamente o modelo RSM apresentou o melhor resultado, seguido do modelo misto (k- $\square$ / RSM). A eficiência de coleta ficou em uma média de aproximadamente $25 \%$ para os três casos. Este valor comprova os dados da literatura: como o diâmetro das partículas foi menor do que o diâmetro de corte do equipamento, a eficiência de coleta foi menor que 50 \%.

Para a figura 11, o modelo RSM conseguiu retratar todo o movimento rotacional do interior do ciclone. Para este caso o diâmetro de corte das partículas está entre o valor do diâmetro de corte do equipamento, dessa forma a eficiência de coleta média para os três casos ficou em torno de $75 \%$. Para o modelo RSM esta porcentagem atingiu $84 \%$, comprovando, através das figuras 10 e 11, uma maior quantidade de partículas retida na caixa coletora. É possível notar ainda que as partículas não coletadas encontram-se abaixo do diâmetro de corte.

\section{CONSIDERAÇÕES FINAIS}

As características indispensáveis ao comportamento fluidodinâmico de ciclones foram bem representadas através de técnicas de fluidodinâmica computacional (CFD), de modo a obterem-se os principais parâmetros de um ciclone da família Lapple.

Entre os modelos de turbulência adotados na realização das simulações, aquele que apresentou os melhores resultados foi o modelo RSM. De fato este modelo é bem mais útil do que o modelo k- $\square$ que, como descrito, não consegue representar de forma eficaz tudo o que ocorre no interior do ciclone. Tanto os perfis de velocidade quanto os de pressão dos modelos RSM foram superiores ao k- $\square$. O modelo misto utilizado também não possui grande utilidade, pois seu desempenho também foi inferior ao RSM. De forma que o modelo RSM é o modelo que deve ser adotado em simulações em caso de dispensa de ensaios experimentais.

Quanto aos resultados obtidos na simulação, estes foram bem condizentes com os dados disponíveis na literatura. As diferenças ocorridas podem ser explicadas pelo fato de ter sido utilizada uma geometria bidimensional, além do seu refinamento não ter sido o melhor possível. Todos os perfis de velocidade seguiram a mesma tendência dos perfis comparativos. Os perfis axial e tangencial demonstraram o mesmo comportamento descrito por Patterson e Munz (1996) e de acordo com as simulações realizadas por Lacerda (2007). 
Os resultados referentes a trajetórias das partículas no interior do ciclone foram de acordo com os resultados de Meier (1998). As partículas de maior diâmetro tendem a ficar mais próximas às paredes do equipamento, seguindo um fluxo descendente, vindo posteriormente a se depositar na caixa coletora; as partículas de menor diâmetro acabam sendo arrastadas juntamente com o fluido para a saída (overflow) do aparelho. Além disso, foi possível observar, através destas simulações, as regiões de reversão do escoamento e as zonas de recirculação. A eficiência de coleta também foi observada de forma eficaz através do modelo probabilístico de Rosin-Rammler para a distribuição granulométrica das partículas. Para partículas acima do diâmetro de corte (dpc), que neste caso foi de $2.45 \mu \mathrm{m}$, houve uma eficiência acima de $50 \%$ e, para partículas abaixo do diâmetro de corte, abaixo de $50 \%$, assim como na literatura.

Fica claro que o uso de CFD torna muito mais fáceis e econômicos os estudos de apareIhos de difícil visualização de fenômenos internos. Esta ferramenta tende a se aperfeiçoar e estar presente dentro da maior parte das empresas e indústrias que visem aprimorar seus processos de produção e de tratamento de resíduos, contribuindo não só para a economia de energia, mas com a diminuição das emissões de partículas e de resíduos para o meio ambiente, além de diminuir expressamente os custos com laboratórios para a realização de experimentos.

\section{REFERÊNCIAS}

BERNARDO, S. Estudo dos escoamentos gasoso e gás-sólido em ciclones pela aplicação de técnicas de fluidodinâmica computacional (CFD). 2005. 266 f. Tese (Doutorado) - Faculdade de Engenharia Química, Universidade Estadual de Campinas, Campinas, 2005.

BOYSANT, F.; AYERS, W. H.; SWITHENBANK, J. A fundamental mathematical modelling approach to cyclone design. Institution of Chemical Engineers, v. 60, p. 222-230, 1982.

CARVALHO, C. M. C. et al. Estudo de escoamentos em ciclones lapple utilizando técnicas da fluidodinâmica computacional (CFD). In: CONGRESSO NACIONAL DE ENGENHARIA MECÂNICA, 7., 2012, São Luís. Anais... São Luís, 2012. 1 CD-ROM.

CICLONES industriais. 2012. Disponível em: <http://engquimicasantossp.blogspot.com. br/2012/07/ciclones-industriais_22.html>. Acesso em: 20 dez. 2012.

DIAS, D. B. Avaliação de técnicas de fluidodinâmica computacional em ciclones.
2009. 126 f. Dissertação (Mestrado) - Faculdade de Engenharia Química, Universidade Estadual de Campinas, Campinas, 2009.

LACERDA, A. F. Estudo dos efeitos das variáveis geométricas no desempenho de ciclones convencionais e filtrantes. 2007. 138f. Tese (Doutorado) - Faculdade de Engenharia Química, Universidade Federal de Uberlândia, Uberlândia, 2007.

LAPPLE, C.E. Process use many collector types. Chemical Engineering, p.144-151, May, 1951.

LUZ, José Aurélio Medeiros da.

Conversibilidade entre distribuições

probabilísticas usadas em modelos de

hidrociclones. Rev. Esc. Minas, v. 58, n. 1, p. 89-93, 2005.

MEIER, H. F. Modelagem fenomenológica e simulação bidimensional de ciclones por técnicas da fluidodinâmica computacional. 1998. 210 f. Tese (Doutorado) - Faculdade de Engenharia Química, Universidade Estadual de Campinas, Campinas 1998.

PATTERSON, P. A; MUNZ, R. J. Gás and particle flow patterns in cyclones at room and elevated temperatures. Canadian J. Chem. Eng., v. 74, p. 213-221, 1996.

PERES, A. P. Técnicas de fluidodinâmica computacional (CFD) aplicadas ao escoamento em ciclones. 2002. Tese (Doutorado) - Faculdade de Engenharia Química, Universidade Estadual de Campinas, Campinas, 2002.

RAMIREZ, M. V. Simulação da perda de carga e da eficiência de coleta em ciclones através da fluidodinâmica computacional (CFD). 2009. 179 f. Dissertação (Mestrado) - Universidade Federal de São Carlos, São Carlos, 2009.

SHEPHERD, C. B.; LAPPLE, C. E. Flow pattern and pressure drop in cyclone dust collectors. Ind. Engineering Chemistry, v. 31, n. 8, p. 972-983, 1939.

VIEIRA, L. G. M. Otimização dos Processos de Separação em Hidrociclones Filtrantes. 2006. Tese (Doutorado) - Faculdade de Engenharia Química, Universidade Federal de Uberlândia, Uberlândia, 2006.

ZHOU, L. X.; SOO, S. L. Gás-solid flow and collection of solid in a cyclone separator. Powder Technology, v. 63, p. 45-53, 1990.

ZHOU, L. X. Theory and numerical modeling of turbulent gas-particle flows and combustion. Beijing: Science Press, 1993. 